\title{
Facial Wrinkles Detection Techniques and its Application
}

\author{
Ashwini Mawale \\ ME Computer (engineering), \\ Dr.D.Y.Patil Institute of Engineering and \\ Technology, Pimpri, Pune-411018. \\ Savitribai Phule Pune University, India
}

\author{
Archana Chaugule \\ Department of Computer Engineering, \\ Dr.D.Y.Patil Institute of Engineering and \\ Technology, Pimpri, Pune-411018. \\ Savitribai Phule Pune University, India
}

\begin{abstract}
Face recognition is process of identifying or verifying individual person by their face. One of the most important sources of the information is human face which can be intended for personal verification and identification of individual person. Wrinkles play an essential role in age estimation. They have been commonly used in applications, such as face age estimation, facial retouching and facial expression recognition. Facial wrinkles present 3D form of skin and appear as skillful discontinuities or cracks in surrounding skin texture. There are different techniques present for facial wrinkles detection. This paper presents the study and review of various techniques used in wrinkle detection. This paper is motivated by need of fast and robust algorithm for detection and classification of human age and facial retouching.
\end{abstract}

\section{Keywords}

Age Classification, Face Recognition, Face Detection methods, Feature Extraction techniques, Wrinkle Detection.

\section{INTRODUCTION}

Recently, face recognition is playing a vital role specifically in the field of commercial, banking, and social area. Human face has numerous features hidden in it which can be used for determining the identity of the person, age of the person, gender of the person. Wrinkles and fine lines are important facial features appear in most aging faces. A correct imagebased analysis play important role in raised aging applications such as age recognition, age simulation and estimation across aging [1-8]. Numerous techniques are present to determine the human age and it has so many real world applications such as vending machines, entertainment, biometrics, surveillance monitoring and cosmetology.

Realistic facial expression synthesis has been one of the most interesting yet difficult problems in computer graphics. Different approaches are present in past to detect facial expression [9-11] by using wrinkles detection. Recognition of human facial expressions is important in man and machine interface of the systems. Generally emotion recognizing from face is very difficult and complex task because distinction in expressing and observing emotions. Wrinkles play very useful role in facial expression recognition and facial analysis.

Another application of Wrinkles detection is facial retouching. Contrast enhancement Techniques are used for beautifying human images which effects highlighting and shading. A nonlinear digital filter bank system is present, which removes objectionable skin components, such as spots and wrinkles, to make the skin look beautified and smoothed. Edge enhancement being used in this system to clear the skin [12][13].In another paper present, localized wrinkles were removed via a constrained texture synthesis technique in image inpainting [14].

As wrinkles are the most important features of aging, the antiaging market to treat facial wrinkles is growing fast. Wrinkles and fine lines are important facial features present in most aging faces. An accurate image-based analysis of these features can play an important role in relevant aging applications. In market cosmetic companies produces various anti-wrinkle creams, and dermatological companies invest in wrinkle filler injections.

While the wrinkles are easily distinguishable by the human eyes, it is a challenging task for image processing to detect them automatically. First, the wrinkles reveal distinctive shapes according to ethnic group, gender, age, and personal life style. Moreover, they are observed as subtle features that highly depend on acquisition environment. In this paper, survey various techniques to detect wrinkle from the image.

\section{LITERATURE SURVEY}

Y. Fu et.al [1] describes that, human age, as an important personal an identifying characteristics, can be directly assumed by separate patterns rising from the facial aspects. Derived from quick advances in machine vision and computer graphics, computer-based age estimation and age synthesis via faces have become specifically preferred topics because of their emerging real-world applications security control and surveillance monitoring, electronic customer relationship management, such as forensic art, entertainment, biometrics, and cosmetology. Because of their complexity and particularity, age synthesis and age estimation are attractive but difficult to computer-based application system designers. Also present models and algorithms designing face aging databases collection, System performances evaluation with valid protocols.

Sung Eun, et al. [2] present an age estimation method combining a hierarchical classifier and hybrid features. In advance, new hierarchical age estimation methods, as well as feature extraction methods for wrinkles and skin, are present. The wrinkle features are extracted by a region specific Gabor filter set and the LBP method is used for the skin features extraction. The hierarchical age estimation is designed to reduce age classification error of boundary data.

N. Ramanathan et.al [3], proposed a twofold approach for modeling facial aging specifically in adults. In first part, author introduced a shape transformation model which captures the delicate deformations facial features with age. In second part, an image gradient based texture transformation function that characterizes facial wrinkles and other skin artifacts often observed. 
K. Luu et.al [6] describes the local and holistic facial features for determining the age of the person based on the characteristics of human craniofacial development. They used combined features that roughly classify a face as young or adult. Features are extracted using two techniques namely AAM and LTP. Active Appearance Model (AAM) linear encoding is used to produce holistic feature and Local Ternary Patterns (LTP) is used to extract local features.

Y.H.Kwon, et.al [8] presents a study of age classification from facial images. In this method input image is distinguished into three age groups such as babies, young and adults. Feature extracted from input images are categorized into two parts. Initial step features are eyes, nose, mouth and chin. Detection and measurement of wrinkles using a wrinkle geography map is secondary step of feature analysis. The problem of varying orientation of the face needs to address. The ratio of computations is easy.

Nazre Batool et.al [14] an algorithm based on the merging of texture orientation fields and Gabor features. Markov field modeling (MRF) is proposed for detect wrinkles and other impurities in the surrounding skin and exemplar-based texture synthesis is proposed to fill the gaps of irregular shapes.

While the wrinkles are easily distinguishable to human eyes, it is a challenging task for computer vision systems to detect them automatically. First, the wrinkles exhibit distinctive shapes according to ethnic group, gender, age, and personal life style. Moreover, they are observed as subtle features that highly depend on acquisition environment. Batool et.al [16] proposed a stochastic wrinkle detection method based on marked point process (MPP). They employed a second derivative linear filter to extract line structures from an image, and penalized an overlap of wrinkle segments. However, their solution strongly depends on the initial condition, and fails to detect complex patterns of wrinkles.

Nazre Batool et.al [17] proposed a method to detect an arbitrary shape of wrinkles, such as a set of line segments, where every segment is distinct by its length and orientation. Also present a probability density of wrinkle model which take advantage of geometric properties and local edge profile of wrinkles. To improve the probability density of wrinkle model, and present reversible jump Markov chain Monte Carlo sampler with delayed rejection.

$\mathrm{Ng}$ et.al [18] proposed a new method, a Hybrid Hessian Filter (HHF). As different parts of the face shows different types of wrinkles. It may lead false detection of wrinkles. Vertical lines are present on the forehead, such as lighting effects and hairs. So author proposed new method Hybrid Hessian Filter (HHF) to deal with this problem. HHF composed of Hessian Matrix and the directional gradient. This is simple method, However, It increases the localization result of wrinkles. The results are compared with Cula method (CLM) and Frangi filter, HHF gives the best result compared to JSI. This method is capable to detect the coarse and medium wrinkles, but not suitable for fine wrinkles.

\section{APPLICATIONS OF FACIAL WRINKLE DETECTION}

Wrinkles detection has been generally used in applications, such as facial expression recognition, face age estimation and facial retouching. (Table 1) shows work done in different applications of wrinkles detection.

Table.1 Applications of Facial Wrinkles Detection

\begin{tabular}{|l|l|l|}
\hline $\begin{array}{l}\text { Age Estimation, age } \\
\text { Synthesis, Age } \\
\text { Simulation }\end{array}$ & $\begin{array}{l}\text { Facial } \\
\text { Expression } \\
\text { Recognition }\end{array}$ & $\begin{array}{l}\text { Facial } \\
\text { Retouching }\end{array}$ \\
\hline $\begin{array}{l}{[1],[2],[3],[4],[5],[6],} \\
{[7],} \\
{[8],[20],[22]}\end{array}$ & {$[9],[10],[11]$} & {$[12],[13],[14]$} \\
\hline
\end{tabular}

\section{RELATED WORKS}

Table.1 Related Works in Wrinkles Detection

\begin{tabular}{|c|c|c|c|}
\hline Ref No & Algorithm/methods & Advantages & Disadvantages \\
\hline 2 & $\begin{array}{c}\text { Gabor filter, local binary } \\
\text { pattern method. }\end{array}$ & $\begin{array}{c}\text { robust to noise such as } \\
\text { shadows, mustache and } \\
\text { hair. }\end{array}$ & $\begin{array}{c}\text { The execution result is } \\
\text { dependent on AAM fitting } \\
\text { result. }\end{array}$ \\
\hline 4 & $\begin{array}{c}\text { Author proposed a } \\
\text { twofold approach for } \\
\text { modeling facial aging } \\
\text { specifically in adults. }\end{array}$ & $\begin{array}{c}\text { Advantages offered by } \\
\text { proposed model are facial } \\
\text { growth statistics, account } \\
\text { for gender-based and } \\
\text { ethnicity-based facial } \\
\text { growth patterns, account for } \\
\text { weight loss/gain. }\end{array}$ & $\begin{array}{c}\text { The proposed facial aging } \\
\text { model cannot account for } \\
\text { facial hair and hence cannot } \\
\text { address hair loss. }\end{array}$ \\
\hline 6 & $\begin{array}{c}\text { combined the local and } \\
\text { holistic facial features for } \\
\text { determining the age of } \\
\text { the person based on the } \\
\text { characteristics of human } \\
\text { craniofacial development }\end{array}$ & $\begin{array}{c}\text { The highest accuracy rates } \\
\text { and efficacy are achieved } \\
\text { by using proposed scheme. }\end{array}$ & $\begin{array}{c}\text { In the holistic approach whole } \\
\text { face region is taken into } \\
\text { account as input data to the } \\
\text { system }\end{array}$ \\
\hline 16 & Marked point process & Representation of wrinkles & depends on the initial \\
\hline
\end{tabular}




\begin{tabular}{|c|c|c|c|}
\hline & $\begin{array}{l}\text { (MPP), reversible jump } \\
\text { Markov chain Monte } \\
\text { Carlo } \\
\text { sampler(RJMCMC). }\end{array}$ & as curve patterns. & $\begin{array}{l}\text { condition, and fails to detect } \\
\text { complex patterns of wrinkles }\end{array}$ \\
\hline 17 & Marked point process & $\begin{array}{l}\text { detects facial wrinkles more } \\
\text { accurately than [16] }\end{array}$ & $\begin{array}{c}\text { False positive values does not } \\
\text { consider here }\end{array}$ \\
\hline 18 & Hybrid Hessian Filter & $\begin{array}{l}\text { Increases localization } \\
\text { result. And reduces false } \\
\text { positive value such as hairs } \\
\text { and light effect. }\end{array}$ & $\begin{array}{l}\text { This method able to detect } \\
\text { medium and coarse wrinkles } \\
\text { but not suitable for fine } \\
\text { wrinkles. }\end{array}$ \\
\hline 19 & $\begin{array}{l}\text { Hessian Line } \\
\text { Tracking(HLT) }\end{array}$ & $\begin{array}{c}\text { Here wrinkles are detected } \\
\text { in curve and valley pattern. } \\
\text { Fine wrinkles detection are } \\
\text { done. }\end{array}$ & $\begin{array}{l}\text { Strongly detector for forehead } \\
\text { wrinkles detection. Wrinkles } \\
\text { detection is not more accurate } \\
\text { in other part of face. }\end{array}$ \\
\hline 20 & Canny edge detector & Detect the boundaries only & $\begin{array}{l}\text { Used for detection of } \\
\text { boundaries only }\end{array}$ \\
\hline 22 & $\begin{array}{l}\text { Digital Template Hough } \\
\text { Transform (DTHT) }\end{array}$ & $\begin{array}{c}\text { Extract both longer and } \\
\text { shorter wrinkles on the } \\
\text { face. }\end{array}$ & $\begin{array}{l}\text { Only boundary detection is } \\
\text { done. It is not sufficient to } \\
\text { detect wrinkles. }\end{array}$ \\
\hline
\end{tabular}

\section{CONCLUSION}

This paper presents the different techniques for facial wrinkles detection, advantages and disadvantages of existing method/algorithm. Also present the various application of wrinkles detection such as age estimation, age synthesis, age simulation, Facial expression recognition and facial retouching. As per survey, there is strong need of fast and robust algorithm to detect Wrinkles as a curve and improve localization results. And to reduce false positive value caused by very light wrinkles, skin discolorations and bright spots.

\section{REFERENCES}

[1] Fu, Yun, Guodong Guo, and Thomas S. Huang. "Age synthesis and estimation via faces: A survey." Pattern Analysis and Machine Intelligence, IEEE Transactions on 32.11 (2010): 1955-1976.

[2] Choi, Sung Eun, et al. "Age estimation using a hierarchical classifier based on global and local facial features." Pattern Recognition 44.6 (2011): 1262-1281.

[3] Dehshibi, Mohammad Mahdi, and Azam Bastanfard. "A new algorithm for age recognition from facial images." Signal Processing 90.8 (2010): 2431-2444.

[4] N.Ramanathan, R.Chellappa, "Modeling shape and textural variations in aging faces, in:FG,2008,pp.1-8.

[5] Jana, Ranjan, Debaleena Datta, and Rituparna Saha. "Age Estimation from Face Image Using Wrinkle Features." Procedia Computer Science 46 (2015): 17541761.

[6] Luu, Khoa, et al. "Combined local and holistic facial features for age-determination." Control Automation Robotics \& Vision (ICARCV), 2010 11th International Conference on. IEEE, 2010.

[7] Ramanathan, Narayanan, Rama Chellappa, and Soma Biswas. "Computational methods for modeling facial aging: A survey." Journal of Visual Languages \& Computing 20.3 (2009): 131-144.
[8] Y. H. Kwon and N. da Vitoria Lobo, "Age classification from facial images," Comput. Vis. Image Understand., vol. 74, no. 1, pp. 1-21, 1999.

[9] Yin, Lijun, and Anup Basu. "Generating realistic facial expressions with wrinkles for model-based coding." Computer vision and image understanding 84.2 (2001): 201-240.

[10] Huang, Yizhen, Ying Li, and Na Fan. "Robust symbolic dual-view facial expression recognition with skin wrinkles: local versus global approach."Multimedia, IEEE Transactions on 12.6 (2010): 536-543.

[11] Park, Gyu-tae, and Zeungnam Bien. "Fuzzy observer approach to automatic recognition of happiness using facial wrinkle features." Fuzzy Systems Conference Proceedings, 1999. FUZZ-IEEE'99. 1999 IEEE International. Vol. 3. IEEE, 1999.

[12] Ohchi, Shuji, Shinichiro Sumi, and Kaoru Arakawa. "A nonlinear filter system for beautifying facial images with contrast enhancement." Communications and Information Technologies (ISCIT), 2010 International Symposium on. IEEE, 2010.

[13] Arakawa, Kaoru. "Nonlinear digital filters for beautifying facial images in multimedia systems." Circuits and Systems, 2004. ISCAS'04. Proceedings of the 2004 International Symposium on. Vol. 5. IEEE, 2004.

[14] Batool, Nazre, and Rama Chellappa. "Detection and inpainting of facial wrinkles using texture orientation fields and markov random field modeling." Image Processing, IEEE Transactions on 23.9 (2014): 3773 3788.

[15] Batool, Nazre, Sima Taheri, and Rama Chellappa. "Assessment of facial wrinkles as a soft biometrics." Automatic Face and Gesture Recognition (FG), 2013 10th IEEE International Conference and Workshops on. IEEE, 2013.

[16] Batool, Nazre, and Rama Chellappa. "Modeling and detection of wrinkles in aging human faces using marked 
point processes." Computer Vision-ECCV 2012. Workshops and Demonstrations. Springer Berlin Heidelberg, 2012.

[17] Batool, Nazre, and Rama Chellappa. "A Markov point process model for wrinkles in human faces." Image Processing (ICIP), 2012 19th IEEE International Conference on. IEEE, 2012.

[18] Ng, Choon-Ching, et al. "Automatic Wrinkle Detection Using Hybrid Hessian Filter." Computer Vision--ACCV 2014. Springer International Publishing, 2015. 609-622.

[19] Ng, Choon-Ching, et al. "Wrinkle detection using hessian line tracking." (2015).
[20] Ng, Choon-Ching, et al. "An Investigation on Local Wrinkle-based Extractor of Age Estimation."

[21] Bando, Yosuke, Takaaki Kuratate, and Tomoyuki Nishita. "A simple method for modeling wrinkles on human skin." Computer Graphics and Applications, 2002. Proceedings. 10th Pacific Conference on. IEEE, 2002.

[22] Hayashi, Jun-ichiro, et al. "Age and gender estimation based on wrinkle texture and color of facial images." Pattern Recognition, 2002. Proceedings. 16th International Conference on. Vol. 1. IEEE, 2002. 